\title{
Continuous gradient nanoporous film enabled by delayed directional diffusion of solvent and selective swelling
}

Di Tan ${ }^{\dagger}$, Qian $\mathrm{Li}^{\dagger}$, Baisong Yang ${ }^{\dagger}$, Xin Wang ${ }^{\dagger}$, Shiqi Hu${ }^{\dagger}$, Zhengzhi Wang ${ }^{\ddagger}$, Yifeng Lei ${ }^{\dagger}$,

$$
\text { Longjian } \mathrm{Xue}^{\dagger^{*}}
$$

†School of Power and Mechanical Engineering, The Institute of Technological Science, Wuhan University, South Donghu Road 8, 430072, Wuhan, China

Department of Engineering Mechanics, School of Civil Engineering, Wuhan University, South

Donghu Road 8, 430072, Wuhan, China

*email: xuelongjian@whu.edu.cn 


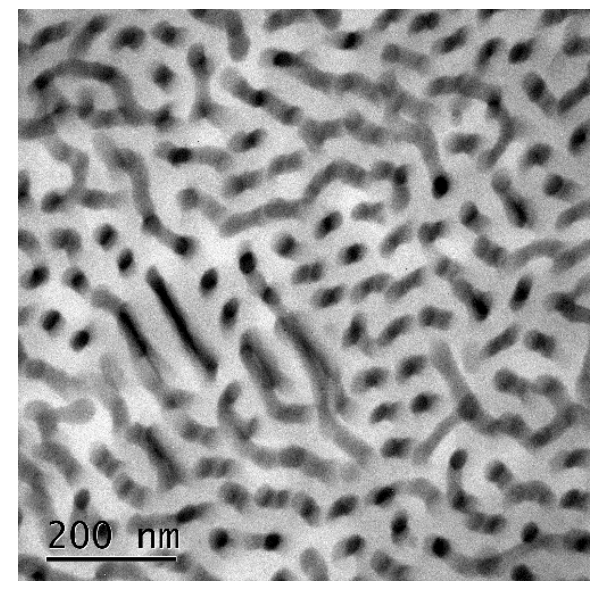

Figure S1. TEM image of microphase separated PS-b-P2VP film, P2VP domains are stained with iodine and appear darker. 
(a)
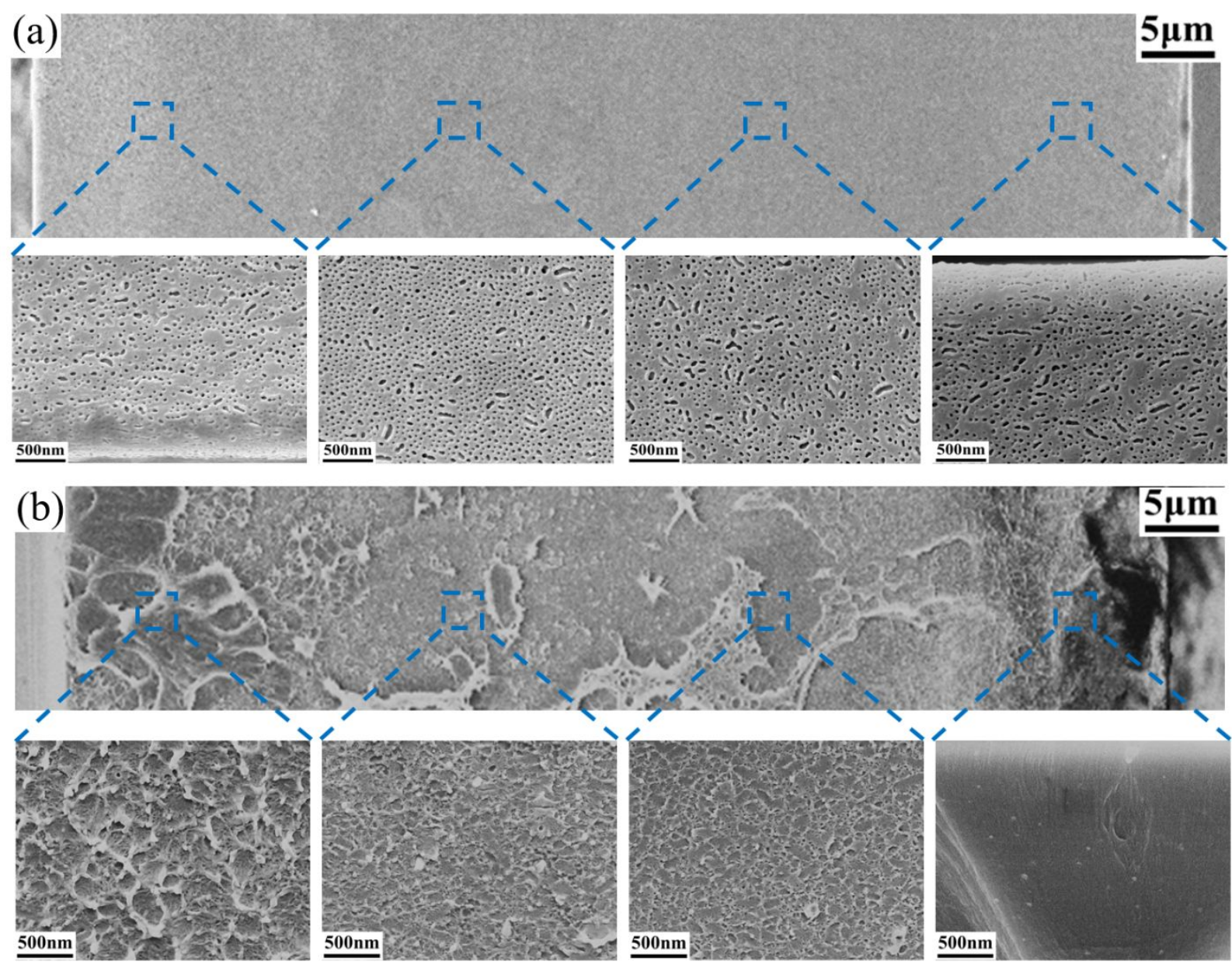

Figure S2. SEM image of cross-section of (a) homogeneous porous PS- $b$-P2VP film and (b) solid PS- $b$-P2VP film. The magnified images in the bottom row in ( $a$ and $b$ ) show the structure at the relative position of $5 \%, 35 \%, 65 \%$ and $95 \%$ of the film thickness. 


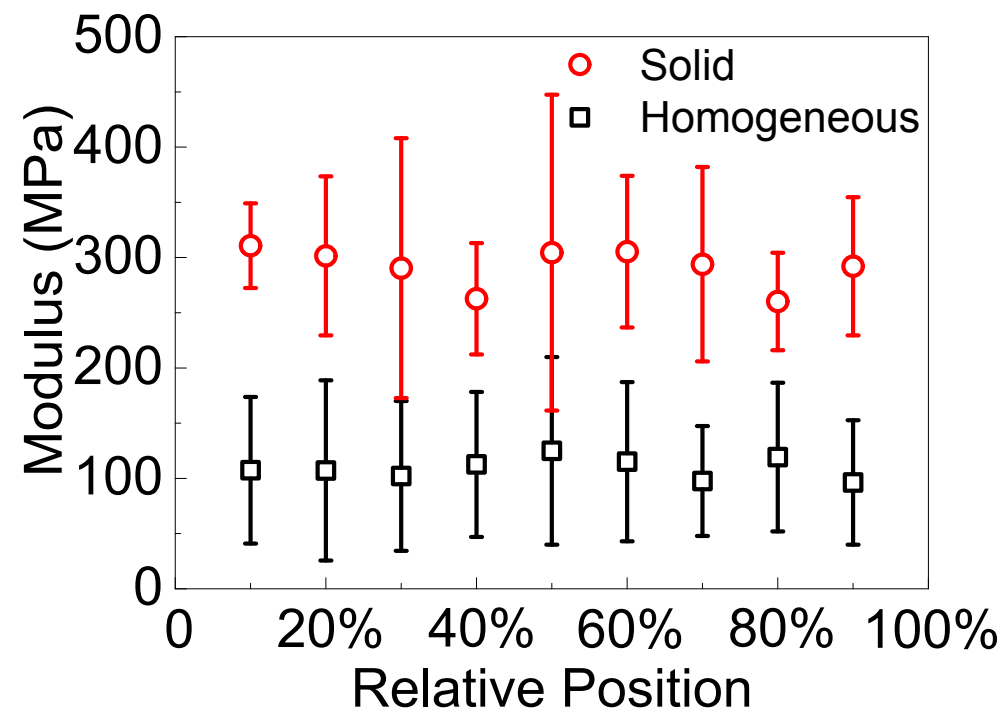

Figure S3. Modulus of the cross-section of solid PS- $b-P 2$ VP film (red spot) and homogeneous porous PS- $b$-P2VP film (black spot). Standard deviations are indicated by error bars. 

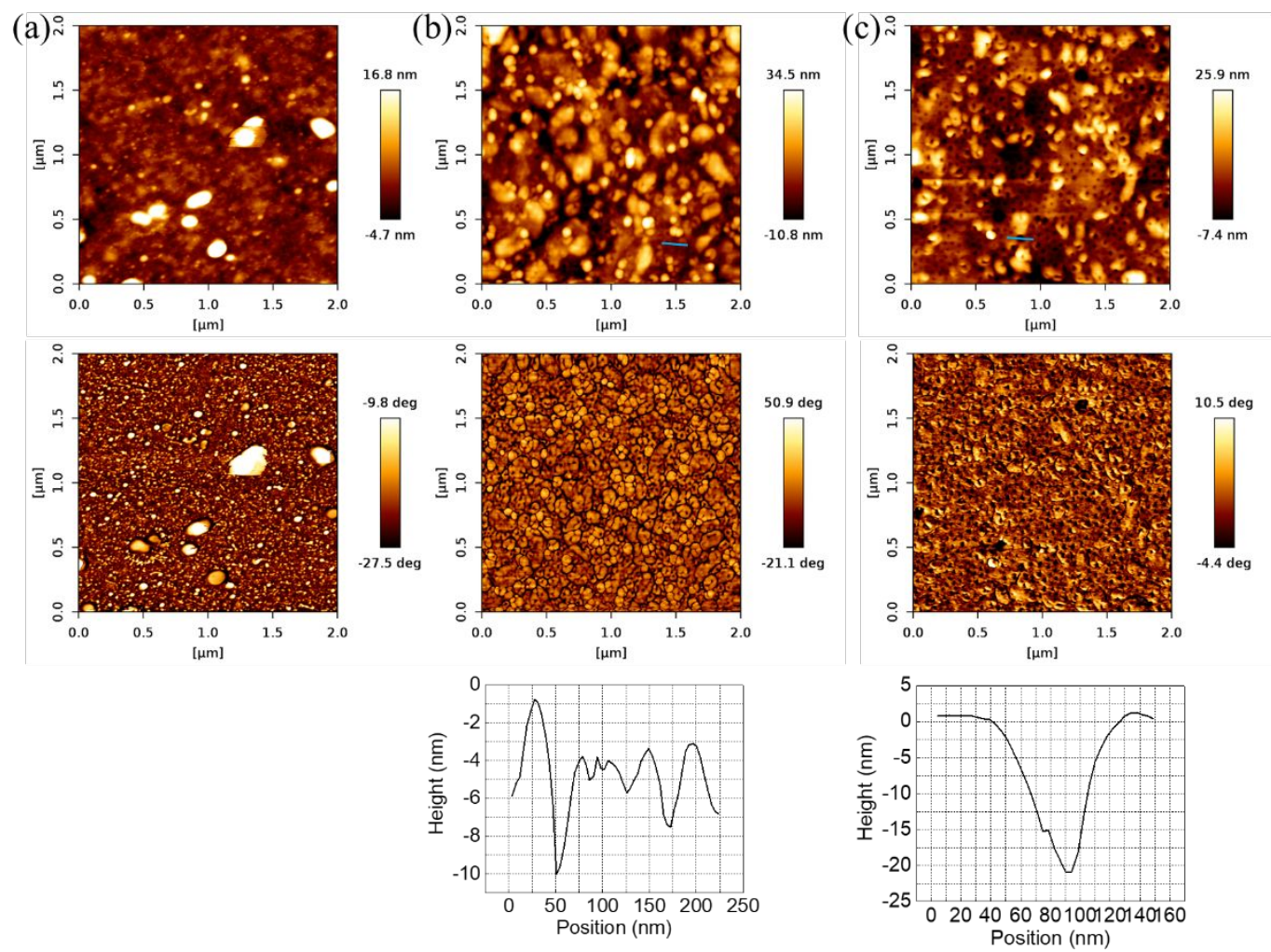

Figure S4. AFM images of PS- $b$-P2VP films treated in ethanol for (a) 0min, (b) $2 \mathrm{~min}$ and (c) $8 \mathrm{~min}$. The first row is the surface morphology. The second row is the phase image and the third row is the profile of structures of first row highlighted by blue lines. 


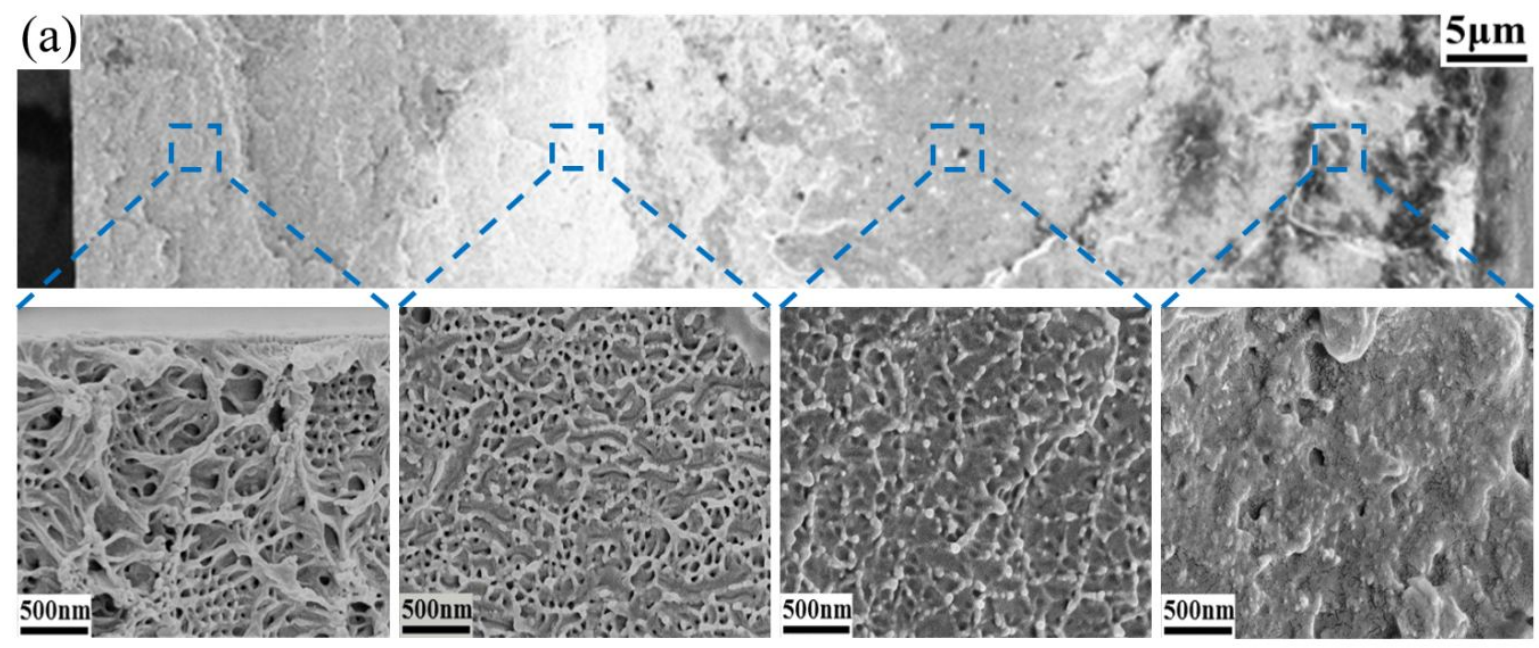

(b)

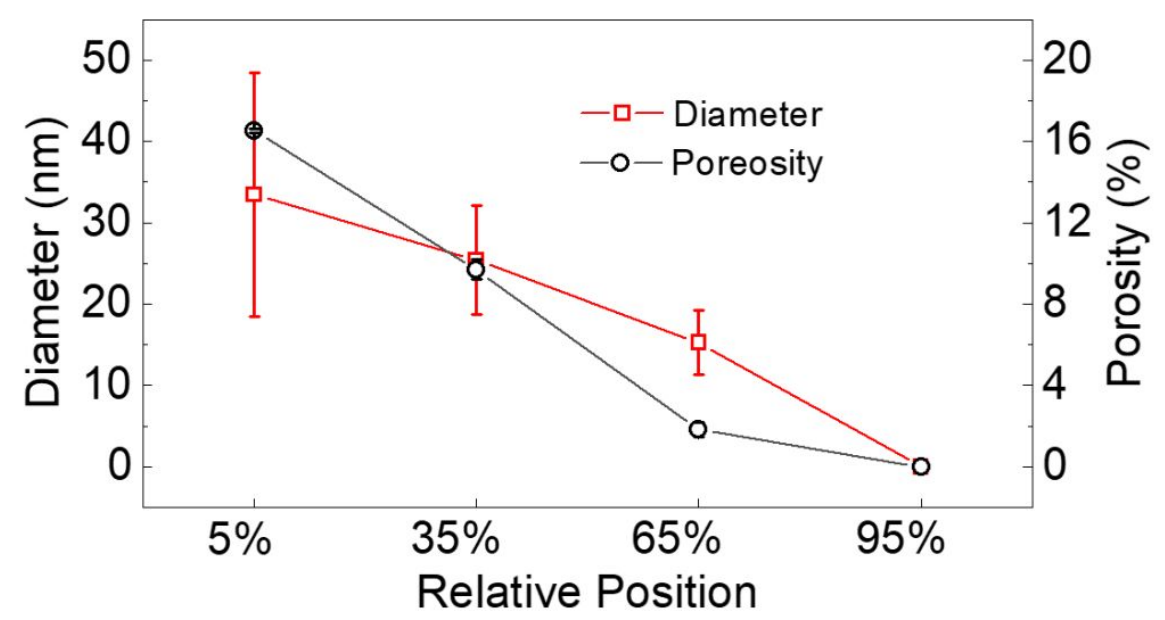

Figure S5. (a) SEM image and (b) pore diameter and porosity of gradient PS- $b-\mathrm{P} 2 \mathrm{VP}$ film induced by PDMS-Al surface pairs in dynamic binary solvent. 

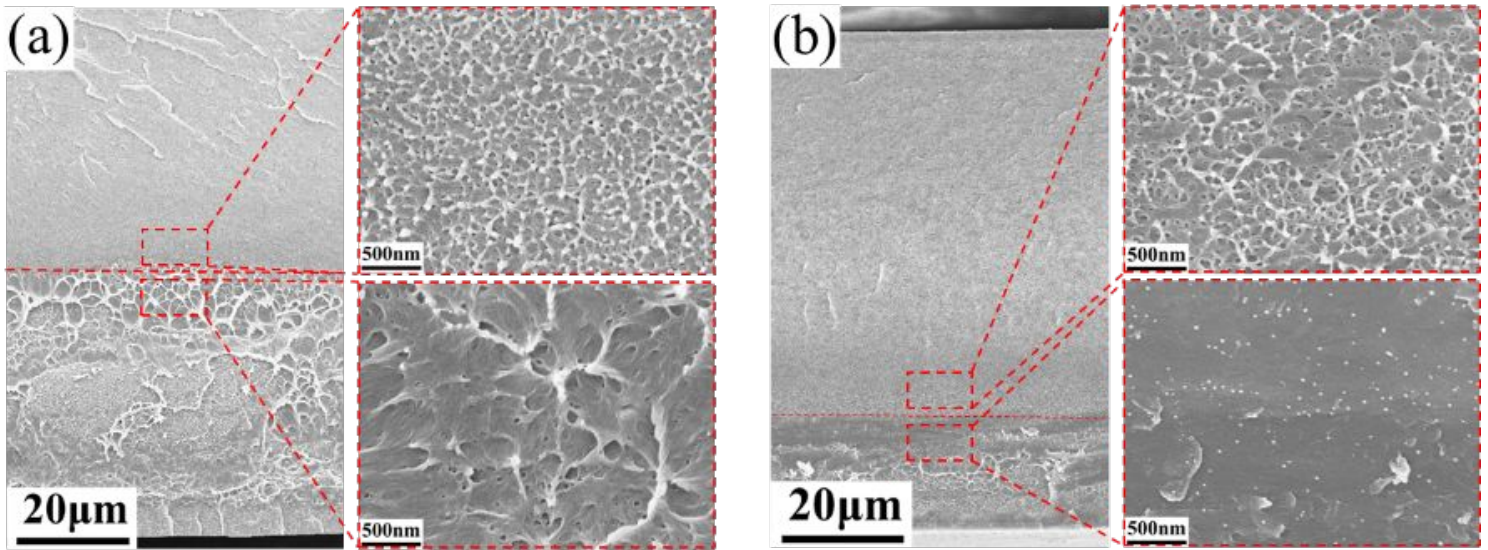

(c)
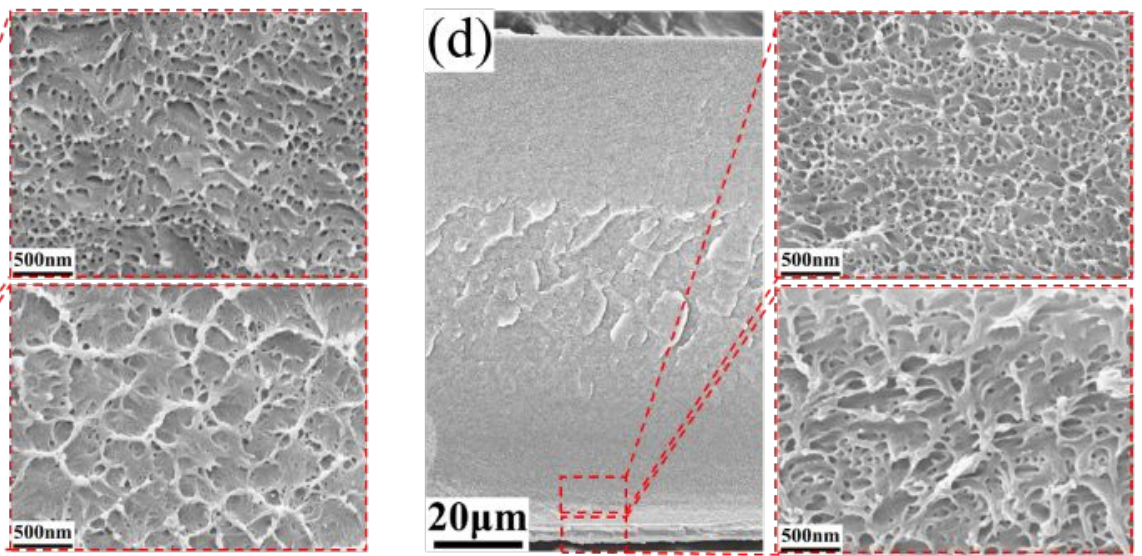

Figure S6. SEM images of diffusion depth in PS- $b$-P2VP film in ethanol at $60^{\circ} \mathrm{C}$ for (a) $5 \mathrm{~min}$, (b) $10 \mathrm{~min}$, (c) $15 \mathrm{~min}$, (d) 20min, respectively. The red dotted line indicates the diffusion front of ethanol. The typical morphology at both sides of red dotted line are shown on the right side of the corresponding figure. 

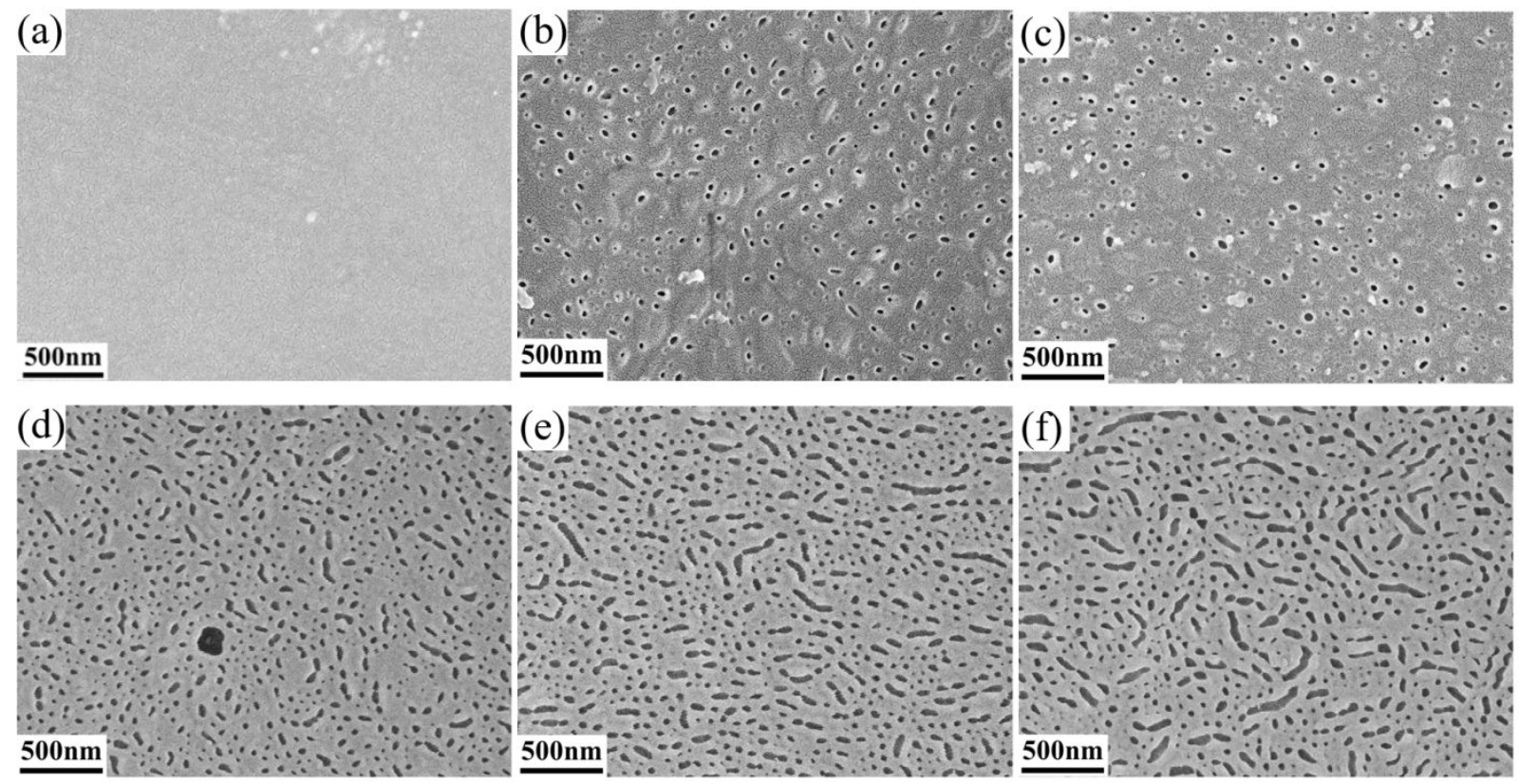

Figure S7. SEM image of porous structure on the film surface with treating time of (a) 0min,

(b) 10min, (c) 30min, (d) 60min, (e) 120min, (f) 240min, respectively. 


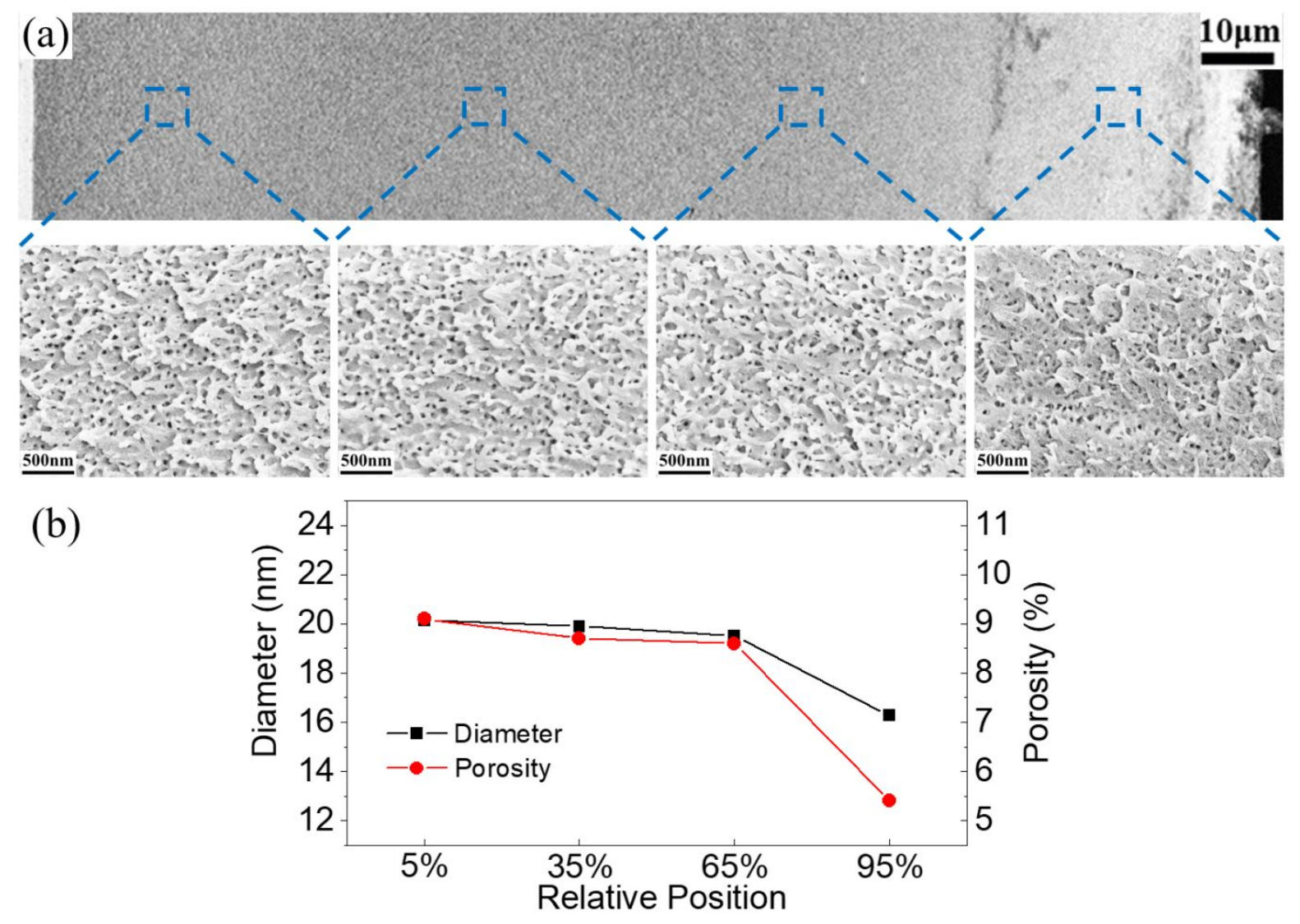

Figure S8. (a) SEM image and (b) pore diameter and porosity of gradient PS- $b-P 2$ VP film induced by PDMS-PDMS surface pairs in pure ethanol for $1 \mathrm{~h}$. The magnified images in (a) show the structure at the relative position of $5 \%, 35 \%, 65 \%$ and $95 \%$ of the film thickness. 

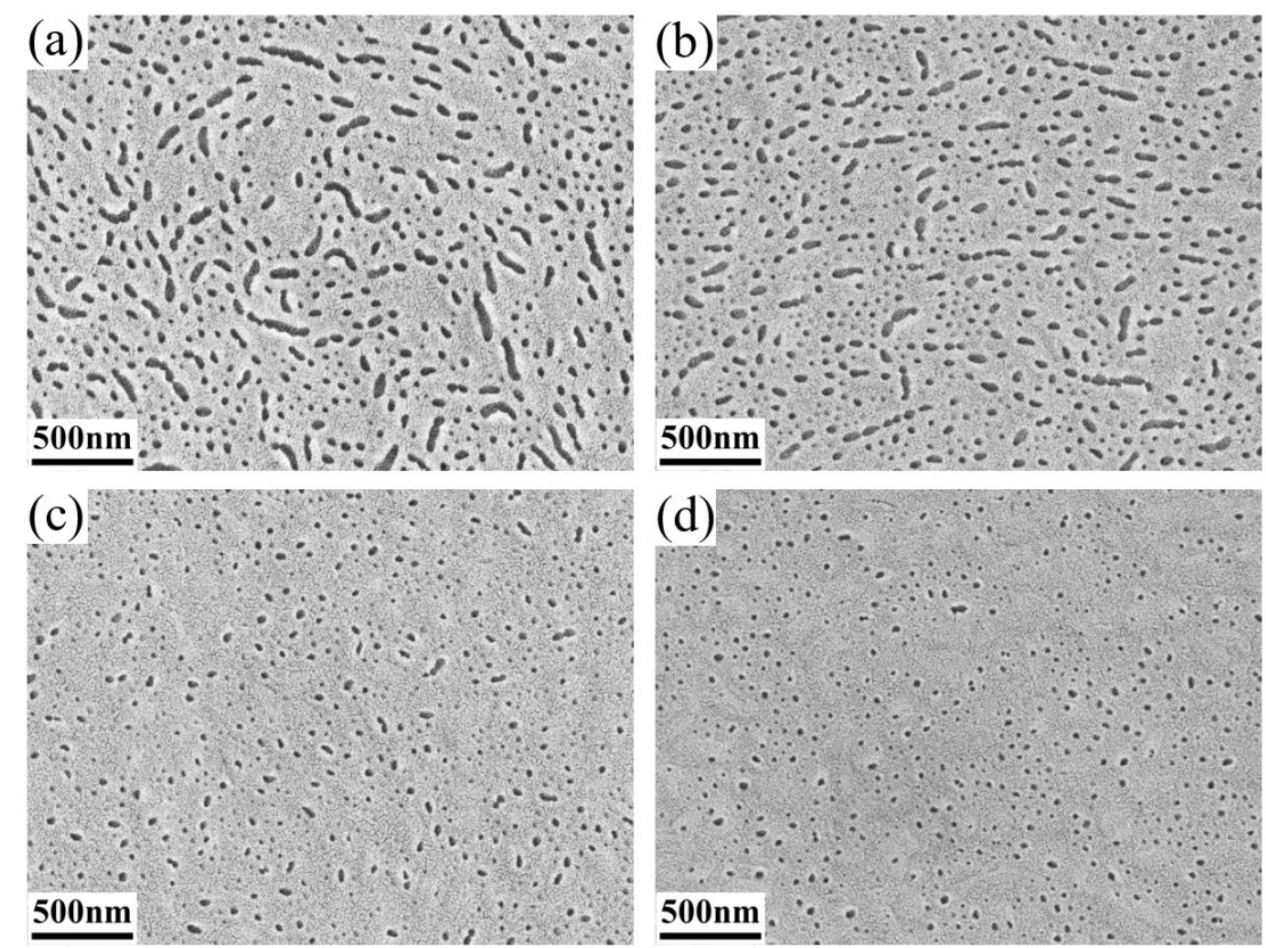

Figure S9. SEM image of porous structures treated in different solvents for 1h: (a) pure ethanol, (b) ethanol $/ \mathrm{H}_{2} \mathrm{O}_{(99: 1)}$, (c) ethanol/ $\mathrm{H}_{2} \mathrm{O}_{(95: 5)}$, (d) ethanol $/ \mathrm{H}_{2} \mathrm{O}_{(90: 10)}$, respectively. 

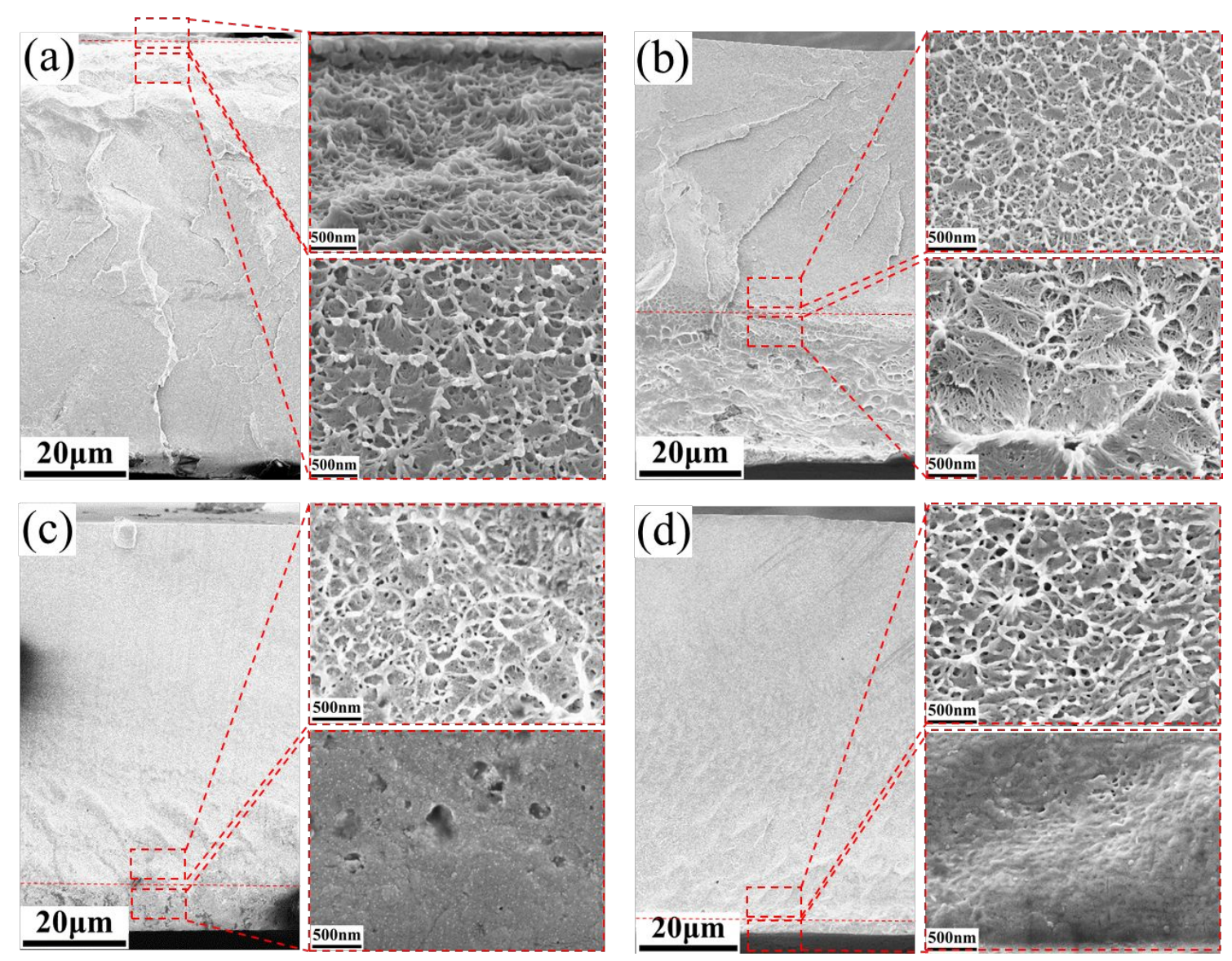

Figure S10. SEM image of diffusion depth in PS- $b$-P2VP film in dynamic binary solvent at 60 ${ }^{\circ} \mathrm{C}$ for (a) $5 \mathrm{~min}$, (b) 20min, (c) 30min, (d) 40min, respectively. The red dotted line indicates the diffusion front of ethanol. The typical morphology at both sides of red dotted line are shown on the right side of the corresponding figure. 